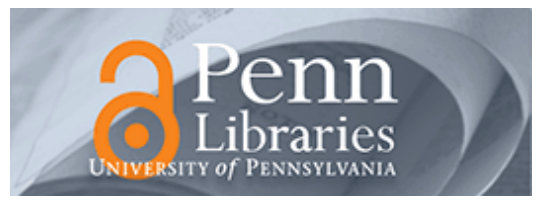

University of Pennsylvania ScholarlyCommons

\title{
The Robustness of Stochastic Switching Networks
}

Po-Ling Loh

University of Pennsylvania

Hongchao Zhou

California Institute of Technology

Jehoshua Bruck

California Institute of Technology

Follow this and additional works at: https://repository.upenn.edu/statistics_papers

Part of the Statistics and Probability Commons

\section{Recommended Citation}

Loh, P., Zhou, H., \& Bruck, J. (2009). The Robustness of Stochastic Switching Networks. Proceedings of the 2009 IEEE International Conference on Symposium on Information Theory, $32066-2070$. Retrieved from https://repository.upenn.edu/statistics_papers/115

This paper is posted at ScholarlyCommons. https://repository.upenn.edu/statistics_papers/115

For more information, please contact repository@pobox.upenn.edu. 


\title{
The Robustness of Stochastic Switching Networks
}

\author{
Abstract \\ Many natural systems, including chemical and biological systems, can be modeled using stochastic \\ switching circuits. These circuits consist of stochastic switches, called pswitches, which operate with a \\ fixed probability of being open or closed. We study the effect caused by introducing an error of size $\in$ to \\ each pswitch in a stochastic circuit. We analyze two constructions--simple series-parallel and general \\ series-parallel circuits--and prove that simple series-parallel circuits are robust to small error \\ perturbations, while general series-parallel circuits are not. Specifically, the total error introduced by \\ perturbations of size less than $\epsilon$ is bounded by a constant multiple of $\epsilon$ in a simple series-parallel circuit, \\ independent of the size of the circuit. However, the same result does not hold in the case of more general \\ series-parallel circuits. In the case of a general stochastic circuit, we prove that the overall error \\ probability is bounded by a linear function of the number of pswitches.

\section{Disciplines} \\ Statistics and Probability
}




\section{The Robustness of Stochastic Switching Networks}

\author{
Po-Ling Loh \\ Department of Mathematics \\ California Institute of Technology \\ Pasadena, CA 91125 \\ Email: loh@caltech.edu
}

\author{
Hongchao Zhou \\ Department of Electrical Engineering \\ California Institute of Technology \\ Pasadena, CA 91125 \\ Email: hzhou@caltech.edu
}

\author{
Jehoshua Bruck \\ Department of Electrical Engineering \\ California Institute of Technology \\ Pasadena, CA 91125 \\ Email: bruck@caltech.edu
}

\begin{abstract}
Many natural systems, including chemical and biological systems, can be modeled using stochastic switching circuits. These circuits consist of stochastic switches, called pswitches, which operate with a fixed probability of being open or closed. We study the effect caused by introducing an error of size $\epsilon$ to each pswitch in a stochastic circuit. We analyze two constructions-simple series-parallel and general series-parallel circuits-and prove that simple series-parallel circuits are robust to small error perturbations, while general series-parallel circuits are not. Specifically, the total error introduced by perturbations of size less than $\epsilon$ is bounded by a constant multiple of $\epsilon$ in a simple series-parallel circuit, independent of the size of the circuit. However, the same result does not hold in the case of more general series-parallel circuits. In the case of a general stochastic circuit, we prove that the overall error probability is bounded by a linear function of the number of pswitches.
\end{abstract}

\section{INTRODUCTION}

Stochastic switching circuits have widespread applications in many fields of modern science, including neuroscience and chemical networks. In neuroscience, stochastic circuits are used to model neural networks, since the propagation of electrical impulses between neurons depends probabilistically on environmental and physical factors [3]. In seeking to model the human brain, it is informative to identify classes of circuits which appear to emulate the behavior of natural systems.

Another application of stochastic switching circuits is found in the framework of chemical reactions [5]. In this context, the probabilities assigned to stochastic switches represent relative concentrations of chemical species after each reaction in a chemical network. Developing algorithms to synthesize stochastic circuits with certain probabilities of being closed may be applied to areas such as drug delivery, where the desired result is a product species with a fixed concentration. Furthermore, since concentrations of chemicals are difficult to control precisely, results on the robustness of stochastic circuits are particularly relevant in the context of chemical networks.

Formally, a stochastic switching circuit $C$ with two terminals is composed of stochastic switches known as pswitches. Each pswitch is assigned a Bernoulli random variable with parameter $0<p<1$, where 1 indicates that the switch is closed and 0 indicates that the switch is open. The set $S$ of probability parameters for the pswitches in a circuit is called the pswitch set. We denote by $P(C)$ the probability that the two terminals of $C$ are connected, and call $P(C)$

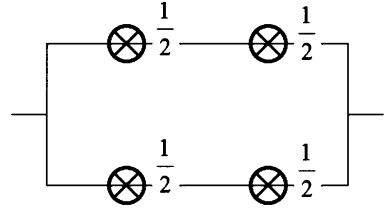

(a) An sp circuit.

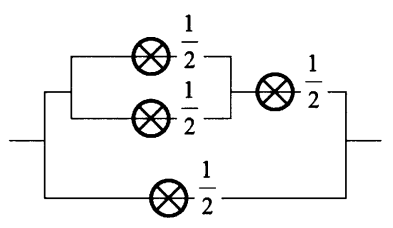

(b) An ssp circuit.
Fig. 1. Examples of sp and ssp circuits. Note that (a) is not ssp.

the probability of $C$. We can realize the probability $x$ using a pswitch set $S$ if and only if there exists a circuit $C$, with pswitch probabilities from $S$, such that $x=P(C)$.

As with resistor circuits [2], we can connect two switching circuits $C_{1}$ and $C_{2}$ in series by connecting one terminal of $C_{1}$ to one terminal of $C_{2}$. Then the probability of the resulting circuit is $p_{1} p_{2}$, where $p_{1}=P\left(C_{1}\right)$ and $p_{2}=P\left(C_{2}\right)$. We can connect $C_{1}$ and $C_{2}$ in parallel by connecting the corresponding terminals of both circuits. The probability of the resulting circuit is $1-\left(1-p_{1}\right)\left(1-p_{2}\right)=p_{1}+p_{2}-p_{1} p_{2}$. A series-parallel (sp) circuit is either (1) a single pswitch, or (2) a series or parallel combination of two sp circuits. Simple series-parallel (ssp) switching circuits are a special case of sp circuits, and are either (1) a single pswitch, or (2) an ssp circuit with an additional pswitch added in series or parallel. See Figure 1.

In [6], the authors proved that any probability of the form $\frac{a}{2^{n}}$, where $0<a<2^{n}$, can be realized with at most $n$ switches, using the pswitch set $S=\left\{\frac{1}{2}\right\}$. However, in natural systems, the states of pswitches may depend on many factors, so we cannot fix their probabilities at specific values.

In this paper, we analyze further properties of stochastic switching circuits, where the probabilities of individual pswitches are taken from a fixed pswitch set, but given an error allowance of $\epsilon$; i.e., the error probabilities of the pswitches are bounded by $\epsilon$. For a stochastic circuit with multiple pswitches, the "error probability" of the circuit is the absolute difference between the probability that the circuit is closed when error probabilities of pswitches are included, and the probability that the circuit is closed when error probabilities are neglected. We show that ssp circuits are robust to small error perturbations, but the error probability of a general sp circuit may be amplified with additional pswitches. In particular, we have the following two theorems: 
Theorem (Robustness of ssp circuits). Given a pswitch set $S$, if the error probability of each pswitch is bounded by $\epsilon$, then the total error probability of an ssp circuit is bounded by $\frac{1}{m} \cdot \epsilon$, where

$$
m=\min \{\min \{S\}, 1-\max \{S\}\} .
$$

Theorem (Unbounded error of sp circuits). Given a pswitch set $S$, if the error probability of each pswitch is bounded by $\epsilon$, then for any $0<x<1$, there exists an sp circuit with error probability close to $x$.

These theorems demonstrate the advantage of ssp circuits over general sp circuits in designing engineering systems. For a general stochastic switching circuit, we also have the following error bound:

Theorem (Error bound of a circuit with $n$ pswitches). Given a pswitch set $S$, if the error probability of each pswitch is bounded by $\epsilon$, then the total error probability of a stochastic switching circuit with $n$ pswitches is bounded by $n \epsilon$.

The remainder of this paper is organized as follows: In Section II, we discuss the error bound for ssp circuits. In Section III, we prove that a similar error bound does not hold in the case of sp circuits. In Section IV, we provide an error bound for general stochastic switching circuits.

\section{Simple Series-Parallel Circuits}

We begin by analyzing the susceptibility of ssp circuits to small error perturbations in individual pswitches. Instead of assigning a pswitch a probability of $p$, the pswitch may be assigned a probability between $p-\epsilon$ and $p+\epsilon$, where $\epsilon$ is a fixed error allowance.

Theorem 1. Given a pswitch set $S$, if the error probability of each pswitch is bounded by $\epsilon$, then the total error probability of an ssp circuit is bounded by $\frac{1}{m} \cdot \epsilon$, where

$$
m=\min \{\min \{S\}, 1-\max \{S\}\} .
$$

Proof: We induct on the number of pswitches. If we have just one pswitch, the result is trivial. Suppose the result holds for $n$ pswitches, and note that for an ssp circuit with $n+1$ pswitches, the last pswitch will either be added in series or in parallel with the first $n$ pswitches. By the induction hypothesis, the circuit constructed from the first $n$ pswitches has probability $p+\epsilon_{1}$ of being closed, where $\epsilon_{1}$ is the error probability introduced by the first $n$ pswitches and $\left|\epsilon_{1}\right| \leq \frac{1}{m} \epsilon$. The $(n+1)^{\text {st }}$ pswitch has probability $t+\epsilon_{2}$ of being closed, where $t \in S$ and $\left|\epsilon_{2}\right| \leq \epsilon$.

If the $(n+1)^{\text {st }}$ pswitch is added in series, then the new circuit (with errors) has probability

$$
\left(p+\epsilon_{1}\right)\left(t+\epsilon_{2}\right)=t p+\epsilon_{2}\left(p+\epsilon_{1}\right)+t \epsilon_{1}
$$

of being closed. Without considering the error probability of each pswitch, the probability of the new circuit is $t p$.

Hence, the overall error probability of the circuit is

$$
e_{1}=\epsilon_{2}\left(p+\epsilon_{1}\right)+t \epsilon_{1} .
$$

By the Triangle Inequality and the induction hypothesis,

$$
\begin{aligned}
\left|e_{1}\right| & \leq\left|\epsilon_{2}\right|\left|\left(p+\epsilon_{1}\right)\right|+t\left|\epsilon_{1}\right| \\
& \leq\left|\epsilon_{2}\right|+t \cdot \frac{1}{m} \epsilon \\
& \leq \frac{m+t}{m} \cdot \epsilon .
\end{aligned}
$$

Note that $m+t \leq(1-\max \{S\})+\max \{S\}=1$, so

$$
\left|e_{1}\right| \leq \frac{1}{m} \cdot \epsilon,
$$

completing the induction.

Similarly, if the $(n+1)^{\text {st }}$ pswitch is added in parallel, then the new circuit (with errors) has probability

$$
\begin{aligned}
& \left(p+\epsilon_{1}\right)+\left(t+\epsilon_{2}\right)-\left(p+\epsilon_{1}\right)\left(t+\epsilon_{2}\right) \\
& =(p+t-t p)+\left(\epsilon_{1}+\epsilon_{2}-t \epsilon_{1}-p \epsilon_{2}-\epsilon_{1} \epsilon_{2}\right)
\end{aligned}
$$

of being closed. Without considering the error probability of each pswitch, the probability that the circuit is closed is $p+$ $t-t p$.

Hence, the overall error probability of the circuit with $n+1$ pswitches is $e_{2}=\epsilon_{1}(1-t)+\epsilon_{2}\left(1-p-\epsilon_{1}\right)$. Again using the induction hypothesis and the Triangle Inequality, we have

$$
\begin{aligned}
\left|e_{2}\right| & \leq(1-t)\left|\epsilon_{1}\right|+\left|\epsilon_{2}\left(1-p-\epsilon_{1}\right)\right| \\
& \leq \frac{1-t}{m} \epsilon+\left|\epsilon_{2}\right| \\
& \leq \frac{1-t+m}{m} \epsilon \\
& \leq \frac{1}{m} \epsilon,
\end{aligned}
$$

since $m=\min \{\min \{S\}, 1-\max \{S\}\} \leq t$. This completes the proof.

As an application, note that if $S=\left\{\frac{1}{2}\right\}$ and each pswitch is given an error allowance of $\epsilon$, then the overall error probability of any ssp circuit with pswitch probabilities from $S$ is bounded by $2 \epsilon$.

\section{General Series-Parallel Circuits}

In the last section, we proved that for a given pswitch set $S$, the overall error probability of an ssp circuit is bounded by a constant multiple of $\epsilon$, where $\epsilon$ is a fixed error allowance for each pswitch. We want to know that whether this property holds for all sp circuits. In this section, we will show that even though the error probability of each pswitch is still bounded by $\epsilon$, the overall error probability of a given circuit may be unbounded as the number of pswitches increases. We have two main results, summarized in Theorems 2 and 3 .

Although the result of Theorem 2 may be deduced from Theorem 3, both theorems provide insight about the nature of robustness in stochastic switching circuits. Theorem 2 is inspired by an iterative construction where two identical circuits are composed on each step. It shows that regardless of the starting circuit (even if it were not sp), we can always build a circuit with an (asymptotic) error probability greater than any constant multiple of $\epsilon$. Rather than using a component-wise 
construction, Theorem 3 constructs a non-ssp circuit which realizes any specified error probability by repeatedly using a single pswitch with error probability $\epsilon$. In this construction, the overall probability that the circuit is closed converges to 1. We are not concerned with the overall probability of the circuits in Theorem 2, since we only analyze the asymptotic behavior of the error as the number of iterations increases.

For the iterative construction, see Figure 2. Beginning with any arbitrary circuit, we calculate the probability that the circuit is closed, and write it as $a+b \epsilon+O\left(\epsilon^{2}\right)$. If $a \geq \frac{1}{2}$, we build the next circuit by taking the previous circuit and putting it in series with itself. If instead $a<\frac{1}{2}$, we take the previous circuit and put it in parallel with itself. Then we iterate the process.

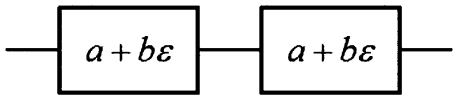

(a) $a \geq \frac{1}{2}$

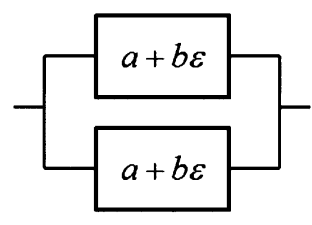

(b) $a<\frac{1}{2}$
Fig. 2. Iterative construction

We see that if $a \geq \frac{1}{2}$, then the probability that the next circuit is closed is

$$
\left(a+b \epsilon+O\left(\epsilon^{2}\right)\right)^{2}=a^{2}+2 a b \epsilon+O\left(\epsilon^{2}\right) .
$$

Hence, the error term is $2 a b \epsilon+O\left(\epsilon^{2}\right)$. Since $a \geq \frac{1}{2}$, we have $2 a b \geq b$, so the coefficient of the $\epsilon$ term grows.

Similarly, if $a<\frac{1}{2}$, then the probability that the next circuit is closed is

$1-\left(1-\left(a+b \epsilon+O\left(\epsilon^{2}\right)\right)\right)^{2}=\left(1-(1-a)^{2}\right)+2(1-a) b \epsilon+O\left(\epsilon^{2}\right)$.

Again, since $a<\frac{1}{2}$, we have $2(1-a) b \geq b$, so the coefficient of $\epsilon$ in the error term grows. We are effectively using a "greedy" approach in this construction, where the coefficient of $\epsilon$ goes from $b$ to $2 \max \{a, 1-a\} b$ on each step.

We now study the rate of growth of the coefficient of $\epsilon$ in the error term. Using simple Matlab code, we generate the graph in Figure 3, which is illustrative of the behavior of the growth of the error coefficient under general initial conditions. (In this graph, we used the initial circuit probability 0.6 , and scaled the coefficient of $\epsilon$ to a starting coefficient of 1.)

In general, the points appear in pairs, with an approximate slope of 0.21 in the linear regression. This corresponds to the error coefficient being multiplied by an average factor of 1.23 on each step.

We now provide a lower bound on the growth of the coefficient of $\epsilon$ in two consecutive steps. We assume $\epsilon$ is small enough that we can ignore the $O\left(\epsilon^{2}\right)$ term.

Lemma 1. Assume the initial circuit is closed with probability $a+\epsilon$. Then the coefficient of $\epsilon$ is multiplied by a factor of at least $\sqrt{2}$ in two consecutive steps.

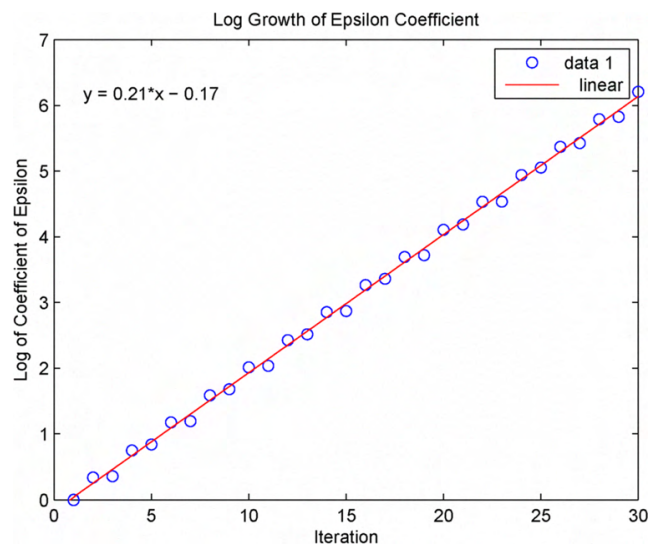

Fig. 3. The growth of the error coefficient.

Proof: Simple algebraic calculations show that for a starting circuit probability of $a+\epsilon$, we have the following four cases:

- $0 \leq a \leq 1-\frac{1}{\sqrt{2}}$. In this case, we put the circuit in parallel with itself, then put the new circuit in parallel: "parallel, followed by parallel."

- $1-\frac{1}{\sqrt{2}} \leq a \leq \frac{1}{2}$. In this case, we have "parallel, followed by series."

- $\frac{1}{2} \leq a \leq \frac{1}{\sqrt{2}}$. In this case, we have "series, followed by parallel."

- $\frac{1}{\sqrt{2}} \leq a \leq 1$. In this case, we have "series, followed by series."

After two steps, the first case yields the error coefficient $4(1-a)^{3}$, the second case yields $4 a(1-a)(2-a)$, the third case yields $4 a\left(1-a^{2}\right)$, and the fourth case yields $4 a^{3}$. So the error coefficient $b$ can be written as

$$
b=\left\{\begin{array}{cc}
4(1-a)^{3}, & 0 \leq a \leq 1-\frac{1}{\sqrt{2}} \\
4 a(1-a)(2-a), & 1-\frac{1}{\sqrt{2}} \leq a \leq \frac{1}{2} \\
4 a\left(1-a^{2}\right), & \frac{1}{2} \leq a \leq \frac{1}{\sqrt{2}} \\
4 a^{3}, & \frac{1}{\sqrt{2}} \leq a \leq 1 .
\end{array}\right.
$$

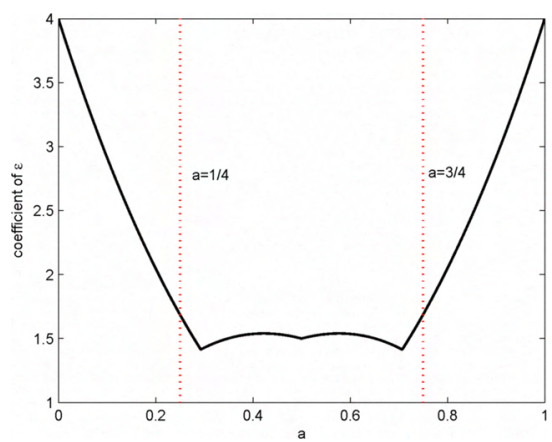

Fig. 4. The growth of the error coefficient of $\epsilon$ as a function of $a$, after two steps.

We graph the growth of the error coefficient in Figure 4, 
and we can check algebraically that the absolute minimum of $\sqrt{2}$ occurs at $1 / \sqrt{2} \approx .707$ and $1-1 / \sqrt{2} \approx .293$.

This proves the unboundedness theorem presented in the introduction:

Theorem 2. Given a pswitch set $S$, if the error probability of each pswitch is bounded by $\epsilon(\epsilon \rightarrow 0)$, then there exists no constant $c$ such that the error probability of any sp circuit with pswitches from $S$ is bounded by $c \epsilon$.

One may conjecture from the preceding discussion that the greedy procedure will always yield the largest error coefficient after an arbitrary number of steps, since we have seen this to be true after two consecutive steps. However, using a starting probability of $.51+\epsilon$, the greedy algorithm does not yield a maximum error coefficient on the third step. Indeed, the greedy algorithm provides the following series of terms:

$$
.51+\epsilon, .26+1.02 \epsilon, .45+1.51 \epsilon, .70+1.65 \epsilon,
$$

corresponding to the sequence "series, parallel, parallel." However, the sequence "parallel, series, series" yields the terms

$$
.51+\epsilon, .76+.98 \epsilon, .58+1.49 \epsilon, .33+1.72 \epsilon .
$$

Comparing the coefficients of $\epsilon$ in the final terms of each sequence, we see that the non-greedy result actually provides a larger error coefficient, disproving the conjecture. However, we have shown that the greedy algorithm already yields a construction where the error coefficient grows exponentially, so using a non-greedy procedure would merely cause the error coefficient to grow even more rapidly.

We also have an upper bound on the rate of growth of the error coefficient in two steps:

Lemma 2. Assume the initial circuit is closed with probability $a+\epsilon$. After an initial number of steps (at most $\left.\log _{2} \log _{\max \{a, 1-a\}} \frac{3}{4}\right)$, the coefficient of $\epsilon$ is multiplied by a factor of at most $\frac{27}{16} \approx 1.688$ in two consecutive steps.

For the proof of Lemma 2, we refer the reader to the Appendix of [1].

In fact, using a different construction, we can obtain a stronger version of Theorem 2:

Theorem 3. Given a pswitch set $S$, if the error probability of each pswitch is bounded by $\epsilon$, then for any $0<x<1$, there exists an sp circuit with error probability close to $x$.

Proof: Suppose $p \in S$. We construct an sp circuit by taking a string of $n$ pswitches of probability $p$, then connecting $m$ of these strings in parallel, where $m=\left\lfloor-\log x \cdot\left(\frac{1}{p}\right)^{n}\right\rfloor$, as shown in Figure 5.

Clearly, the probability that the circuit will be closed is

$$
1-\left(1-p^{n}\right)^{m} \text {. }
$$

To simplify our computations, we let $m=-\log x \cdot\left(\frac{1}{p}\right)^{n}$. Then

$$
\lim _{n \rightarrow \infty}\left(1-p^{n}\right)^{m}=\lim _{n \rightarrow \infty} e^{m \cdot \log \left(1-p^{n}\right)} .
$$

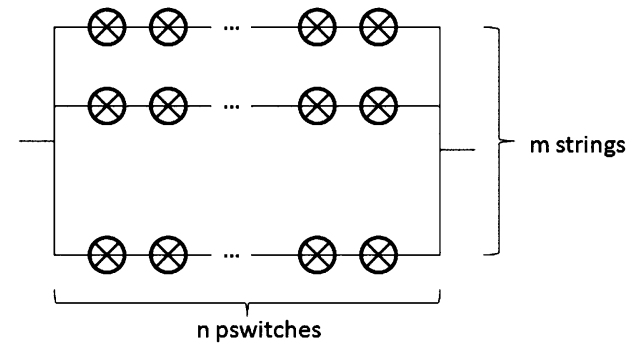

Fig. 5. The construction of a noisy sp circuit.

Note that

$$
\begin{aligned}
\lim _{n \rightarrow \infty} m \cdot \log \left(1-p^{n}\right) & =\lim _{n \rightarrow \infty} \frac{-\log x \cdot \log \left(1-p^{n}\right)}{p^{n}} \\
& =\frac{-\log x \cdot \frac{1}{1-p^{n}} \cdot-p^{n} \log p}{p^{n} \log p} \\
& =\log x .
\end{aligned}
$$

Hence, the probability that the circuit will be closed converges to

$$
1-e^{\log x}=1-x
$$

as $n \rightarrow \infty$.

Now suppose we introduce an error of $\epsilon$ to each pswitch, so the probability that each pswitch in the circuit is closed is $q=p+\epsilon$. Then the probability that the circuit is closed is $1-\left(1-q^{n}\right)^{m}$, and we compute

$$
\begin{aligned}
\lim _{n \rightarrow \infty} m \cdot \log \left(1-q^{n}\right) & =\lim _{n \rightarrow \infty} \frac{-\log x \cdot \log \left(1-q^{n}\right)}{p^{n}} \\
& =\lim _{n \rightarrow \infty} \frac{-\log x \cdot \frac{1}{1-q^{n}} \cdot-q^{n} \log q}{p^{n} \log p} \\
& =\frac{\log x \cdot \log q}{\log p} \cdot \lim _{n \rightarrow \infty}\left(\frac{q}{p}\right)^{n} .
\end{aligned}
$$

Note that since $0<p, q<1$, we have $\frac{\log x \cdot \log q}{\log p}<0$. So for $\epsilon>0$, we have $\frac{q}{p}>1$, implying that the limit is $-\infty$. Hence, the probability that the circuit is closed converges to $1-0=1$.

However, we still need to take into consideration the fact that $m=\left\lfloor-\log x \cdot\left(\frac{1}{p}\right)^{n}\right\rfloor$. (We need to make this modification in order to ensure that $m$ is an integer.) Note that adding the floor function changes $m$ by at most 1 . Then the quantity $\left(1-p^{n}\right)^{m}$ (or $\left(1-q^{n}\right)^{m}$ ) changes by at most a factor of $\left(1-p^{n}\right)$. As $n$ increases, this factor approaches 1 . Hence, we can constrain $n$ such that $\left(1-p^{n}\right)$ is close enough to 1 , and the probabilities that the circuits are closed can still be made arbitrarily close to $1-x$ and 1 .

\section{iV. General Stochastic Switching Circuits}

In this section, we extend our discussion to the case of general stochastic switching circuits. We have the following theorem, which clearly also holds for sp and ssp circuits:

Theorem 4. Given a general stochastic switching circuit with $n$ pswitches taken from a finite pswitch set $S$, if each 
pswitch has error probability bounded by $\epsilon$, then the total error probability of the circuit is bounded by $n \epsilon$.

Proof:

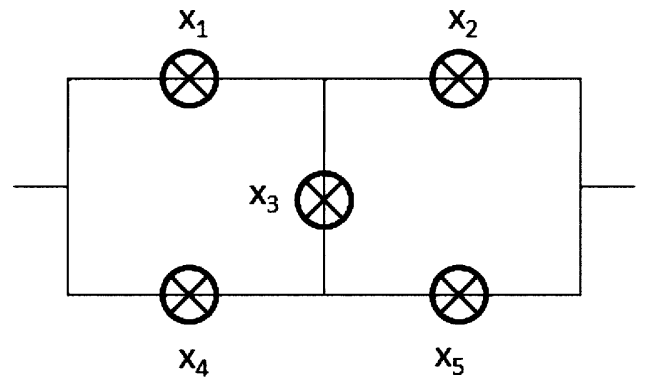

Fig. 6. An example of a general stochastic switching circuit.

We first index all the pswitches in the circuit $C$ as shown in Figure 6 . If $x_{i}$ is the probability that the $i^{\text {th }}$ pswitch is closed, we write $C\left(x_{1}, x_{2}, \ldots, x_{n}\right)$ to denote the $n$-pswitch circuit. For each $i$, we write $x_{i}=a_{i}+\epsilon_{i}$, where $a_{i} \in S$ and $\epsilon_{i}$ is the error probability (so $\left|\epsilon_{i}\right| \leq \epsilon$ ).

Let $P^{(k)}$ denote the probability that $C$ is closed when we only take into account the error probabilities of the first $k$ pswitches; i.e.,

$$
P^{(k)}=P\left(C\left(x_{1}, \ldots, x_{k}, a_{k+1}, \ldots, a_{n}\right)\right) .
$$

The overall error of the circuit can then be written as

$$
\begin{aligned}
E= & P^{(n)}-P^{(0)} \\
= & \left(P^{(n)}-P^{(n-1)}\right)+\left(P^{(n-1)}-P^{(n-2)}\right)+ \\
& \cdots+\left(P^{(1)}-P^{(0)}\right) .
\end{aligned}
$$

We need to prove that $\left|P^{(k)}-P^{(k-1)}\right| \leq \epsilon$ for all $1 \leq k \leq n$. We write

$$
\begin{aligned}
& \left|P^{(k)}-P^{(k-1)}\right| \\
= & \mid P\left(C\left(x_{1}, \ldots, x_{k}, a_{k+1}, \ldots, a_{n}\right)\right) \\
& -P\left(C\left(x_{1}, \ldots, x_{k-1}, a_{k}, \ldots, a_{n}\right)\right) \mid \\
= & \mid x_{k} P\left(C\left(x_{1}, \ldots, x_{k-1}, 1, a_{k+1}, \ldots, a_{n}\right)\right) \\
& +\left(1-x_{k}\right) P\left(C\left(x_{1}, \ldots, x_{k-1}, 0, a_{k+1}, \ldots, a_{n}\right)\right) \\
& -a_{k} P\left(C\left(x_{1}, \ldots, x_{k-1}, 1, a_{k+1}, \ldots, a_{n}\right)\right) \\
& -\left(1-a_{k}\right) P\left(C\left(x_{1}, \ldots, x_{k-1}, 0, a_{k+1}, \ldots, a_{n}\right)\right) \mid \\
= & \mid \epsilon_{k} \cdot\left[P\left(C\left(x_{1}, \ldots, x_{k-1}, 1, a_{k+1}, \ldots, a_{n}\right)\right)\right. \\
& \left.-P\left(C\left(x_{1}, \ldots, x_{k-1}, 0, a_{k+1}, \ldots, a_{n}\right)\right)\right] \mid \\
\leq & \left|\epsilon_{k}\right| \\
\leq & \epsilon .
\end{aligned}
$$

Therefore, we have

$$
\begin{aligned}
E \leq & \left|P^{(n)}-P^{(n-1)}\right|+\left|P^{(n-1)}-P^{(n-2)}\right|+ \\
& \cdots+\left|P^{(1)}-P^{(0)}\right| \\
\leq & n \epsilon,
\end{aligned}
$$

as wanted.
Note that this is the best bound we can afford in the case of a general pswitch set $S$ and any arbitrary $\epsilon$. Indeed, given a value of $n$, choose $p$ close to 1 and $\epsilon<<p$. Putting $n$ pswitches of probability $p-\epsilon$ in series, we have probability

$$
(p-\epsilon)^{n} \approx p^{n}-n p^{n-1} \epsilon
$$

that the circuit is closed. Without errors, the probability of the circuit is $p^{n}$, so the overall error is

$$
n \cdot p^{n-1} \epsilon \text {. }
$$

Choosing $p$ sufficiently close to 1 , we can make the error probability of the circuit arbitrarily close to $n \epsilon$.

\section{CONClusion}

In this paper, we have analyzed the effects caused by small error perturbations in stochastic switching circuits and shown that ssp circuits are robust to small errors of size $\epsilon$, while general sp circuits are not. We have also provided a linear bound for the error in a general stochastic switching circuit, when each pswitch has an error probability of at most $\epsilon$.

Our result on the robustness of ssp circuits supports the hypothesis that ssp circuits provide a better model for biological systems than general sp circuits. This is consistent with the observation that the inductive construction of an ssp circuit resembles the synthesis of natural systems through biological or evolutionary growth. Further directions of research include analyzing the case where the probabilities assigned to the pswitches are not discrete, but continuous (perhaps timedependent probabilities), and considering other systems where the rules of composition for series and parallel are analogous to the rules of composition for electrical circuits.

\section{ACKNOWLEDGMENT}

This work was supported in part by the NSF Expeditions in Computing Program under grant CCF-0832824. The authors would also like to thank the Caltech Summer Undergraduate Research Fellowship (SURF) program for its support in funding this research, and Dan Wilhelm for his comments and suggestions in revising the paper.

\section{REFERENCES}

[1] P. Loh, H. Zhou and J. Bruck, "The Robustness of Stochastic Switching Networks." Appears as Caltech Paradise Electronic Techinical Report at http://www.paradise.caltech.edu/papers/etr092.pdf.

[2] P.A. Macmahon, "The combinations of resistances," The Electrician, vol 28, pp. 601-602, 1892. (Reprinted in: Discr. Appl. Math., 54:225-228, 1994.)

[3] F. Rieke, D. Warland, R.R. van Steveninck, W. Bialek, "Spikes: exploring the neural code," MIT Computational Neuroscience series, 1999.

[4] C. Shannon, "A symbolic analysis of relay and switching circuits," Trans. $A I E E$, vol. 57, pp. 713-723, 1938.

[5] D. Soloveichik, M. Cook, E. Winfree, J. Bruck, "Computation with finite stochastic chemical reaction networks," Natural Computing 7(4), pp. 615$633,2008$.

[6] D. Wilhelm and J. Bruck, "Stochastic switching circuit synthesis," IEEE International Symposium on Information Theory, Toronto, Canada, July 2008. Also appears as Caltech Paradise Electronic Technical Report at http://www.paradise.caltech.edu/papers/etr089.pdf. 